Article

\title{
In Vivo Secretion of $\beta$-Lactamase-Carrying Outer Membrane Vesicles as a Mechanism of $\beta$-Lactam Therapy Failure
}

\author{
Martina Bielaszewska ${ }^{1, *(\mathbb{C})}$, Ondřej Daniel ${ }^{1,2}{ }^{\mathbb{D}}$, Otakar Nyč $^{3}$ and Alexander Mellmann ${ }^{4}(\mathbb{D}$ \\ 1 Centre for Epidemiology and Microbiology, National Institute of Public Health, \\ 10000 Prague, Czech Republic; ondrej.daniel@szu.cz \\ 2 Second Medical Faculty, Charles University, 15006 Prague, Czech Republic \\ 3 Institute of Medical Microbiology, University Hospital Motol, 15006 Prague, Czech Republic; \\ Otakar.Nyc@fnmotol.cz \\ 4 Institute for Hygiene, University Hospital Münster, 48149 Münster, Germany; \\ alexander.mellmann@ukmuenster.de \\ * Correspondence: martina.bielaszewska@szu.cz; Tel.: +420-267082979
}

\section{check for} updates

Citation: Bielaszewska, M.; Daniel, O.; Nyč, O.; Mellmann, A. In Vivo Secretion of $\beta$-Lactamase-Carrying Outer Membrane Vesicles as a Mechanism of $\beta$-Lactam Therapy Failure. Membranes 2021, 11, 806. https://doi.org/10.3390/ membranes11110806

\section{Academic Editors: Ganesh}

Vilas Shelke, Stefania Raimondo and Jaewook Lee

Received: 23 September 2021

Accepted: 18 October 2021

Published: 23 October 2021

Publisher's Note: MDPI stays neutral with regard to jurisdictional claims in published maps and institutional affiliations.

Copyright: (c) 2021 by the authors. Licensee MDPI, Basel, Switzerland. This article is an open access article distributed under the terms and conditions of the Creative Commons Attribution (CC BY) license (https:// creativecommons.org/licenses/by/ $4.0 /)$.

\begin{abstract}
Outer membrane vesicles carrying $\beta$-lactamase ( $\beta$ LOMVs) protect bacteria against $\beta$-lactam antibiotics under experimental conditions, but their protective role during a patient's treatment leading to the therapy failure is unknown. We investigated the role of $\beta$ LOMVs in amoxicillin therapy failure in a patient with group A Streptococcus pyogenes (GAS) pharyngotonsillitis. The patient's throat culture was examined by standard microbiological procedures. Bacterial vesicles were analyzed for $\beta$-lactamase by immunoblot and the nitrocefin assay, and in vivo secretion of $\beta$ LOMVs was detected by electron microscopy. These analyses demonstrated that the patient's throat culture grew, besides amoxicillinsusceptible GAS, an amoxicillin-resistant nontypeable Haemophilus influenzae (NTHi), which secreted $\beta$ LOMVs. Secretion and $\beta$-lactamase activity of NTHi $\beta$ LOMVs were induced by amoxicillin concentrations reached in the tonsils during therapy. The presence of NTHi $\beta$ LOMVs significantly increased the minimal inhibitory concentration of amoxicillin for GAS and thereby protected GAS against bactericidal concentrations of amoxicillin. NTHi $\beta$ LOMVs were identified in the patient's pharyngotonsillar swabs and saliva, demonstrating their secretion in vivo at the site of infection. We conclude that the pathogen protection via $\beta$ LOMVs secreted by the flora colonizing the infection site represents a yet underestimated mechanism of $\beta$-lactam therapy failure that warrants attention in clinical studies.
\end{abstract}

Keywords: group A Streptococcus pyogenes (GAS); pharyngotonsillitis; amoxicillin therapy failure; $\beta$-lactamase-carrying outer membrane vesicles; Haemophilus influenzae; in vivo secretion; GAS protection

\section{Introduction}

Group A Streptococcus pyogenes (GAS) is the most common cause of acute bacterial pharyngotonsillitis, accounting for $20-30 \%$ of cases in children and $5-15 \%$ of cases in adults [1]. GAS is highly susceptible to $\beta$-lactam antibiotics, so penicillin and amoxicillin are the treatments of choice [1]. However, the inabilities of these antibiotics to eradicate GAS from patients with pharyngotonsillitis have been increasingly reported [2]. One cause of the therapy failure is colonization of the pharynx and tonsils by $\beta$-lactamase-producing bacteria such as Moraxella catarrhalis, Haemophilus influenzae, and Staphylococcus aureus that protect GAS against $\beta$-lactam antibiotics [2]. Yet, the mechanisms of this protection are incompletely understood. Here, we investigated the involvement of bacterial outer membrane vesicles (OMVs) in amoxicillin therapy failure.

OMVs are bilayered spherical nanoparticles in size between 10 and $300 \mathrm{~nm}$ produced by Gram-negative bacteria [3-6]. They are formed by a bulging of the bacterial outer membrane followed by fission of the nascent vesicle and its subsequent release from the bacterial cell [6,7]. They mainly contain the outer membrane components and periplasmic proteins [3-6]. OMVs represent a novel, highly efficient bacterial secretion system [5], 
which mediates multiple interbacterial and microbe-host interactions $[3,5,6,8-10]$. They are produced both in vitro and in vivo [3,10-12]. Secretion of OMVs is significantly increased under environmental stress conditions, including those encountered by the bacteria within the host, and enables bacteria to cope with and adapt to this stress [8,13-15]. Notably, exposure of bacteria to particular antibiotics results in increased OMV production [16-19], suggesting that OMVs are involved in the bacterial protection against these drugs. Several mechanisms of the protection have been proposed based on experimental data [18-26]. One of these mechanisms, which occurs in $\beta$-lactamase-producing bacteria, involves production of OMVs that carry various $\beta$-lactamases, thereby protecting susceptible bacteria of the same and other species against $\beta$-lactam antibiotics under in vitro conditions $[18,20,22-26]$. However, the involvement of this mechanism in $\beta$-lactam therapy failure in vivo during patients' treatment remains unknown. We therefore explored the role of $\beta$-lactamase-carrying OMVs secreted in vivo by amoxicillin-resistant Haemophilus influenzae in amoxicillin therapy failure in a patient with GAS pharyngotonsillitis. We demonstrated that these OMVs protected GAS against amoxicillin, which resulted in bacteriological and clinical treatment failure.

\section{Materials and Methods}

\subsection{Patient and Microbiological Examinations}

A 39-year-old man presented with acute pharyngotonsillitis accompanied by a fever up to $39.8^{\circ} \mathrm{C}$. His throat swabs were cultured on blood agar and chocolate agar (Thermo Fisher Scientific, Prague, Czech Republic) and the isolates were identified by standard bacteriological procedures [27] and MALDI-TOF mass spectrometry (Microflex LT, Bruker Daltonics, Bremen, Germany). Antimicrobial susceptibilities were determined by the disc diffusion method (discs from Oxoid, Brno, Czech Republic) and the broth microdilution method according to the Clinical and Laboratory Standards Institute (CLSI) procedures and criteria [28]. The bla $a_{\mathrm{TEM}-1}$ gene was detected by PCR followed by digestion of $600 \mathrm{bp}$ amplicon with MboI (New England Biolabs, Frankfurt am Main, Germany) as described previously [29].

\subsection{Ethical Approval}

The study was conducted according to the guidelines of the Declaration of Helsinki and approved by the Ethics Committee of the National Institute of Public Health, Prague (protocol code EK-SZU/08076/2021), on 1 June 2021. Written informed consent was obtained from the patient.

\subsection{Isolation and Characterization of OMVs Produced by Nontypeable Haemophilus Influenzae (NTHi) Patient's Isolate}

The NTHi patient's isolate was cultured overnight in a brain heart infusion (BHI) broth (Thermo Fisher Scientific, Prague, Czech Republic) supplemented with NAD (nicotinamide adenine dinucleotide) and hemin $(10 \mu \mathrm{g} / \mathrm{mL}$ each) (Sigma-Aldrich, Taufkirchen, Germany). To determine the effect of amoxicillin on OMV production, the medium was supplemented with amoxicillin (Sigma-Aldrich, Taufkirchen, Germany) in concentrations reported in the tonsillar tissue during amoxicillin therapy $(0.17 \mu \mathrm{g} / \mathrm{mL}, 1.1 \mu \mathrm{g} / \mathrm{mL}$, and $3.9 \mu \mathrm{g} / \mathrm{mL}$ ) [30-32]. OMVs were isolated by ultracentrifugation as described previously [33,34]. OMV amounts were determined by nanoparticle tracking analysis [15,35], morphology by electron microscopy after negative staining [34], and protein concentrations with Roti-Nanoquant (Carl Roth, Karlsruhe, Germany) in accordance with the manufacturer's instructions. OMV-associated $\beta$-lactamase was detected by immunoblot [34] with a mouse monoclonal anti- $\beta$-lactamase antibody (Abcam, Cambridge, United Kingdom, Cat\# ab12251, RRID: AB_298974) and an alkaline phosphatase-conjugated goat antimouse IgG (Dianova, Hamburg, Germany, Cat\# 115-055-146, RRID:AB_2338538); signals were developed with NBT/BCIP (nitro blue tetrazolium chloride/5-bromo-4-chloro-3' indolyl phosphate, toluidine salt) substrate (Roche, Mannheim, Germany) and visualized with a Chemi Doc XRS imager (BioRad, Munich, Germany). Localization of $\beta$-lactamase 
within OMVs was determined by the proteinase $\mathrm{K}$ assay, as described previously [22,36]. Briefly, OMVs, either intact or lyzed with 0.1 M EDTA (ethylenediaminetetraacetic acid disodium salt dihydrate) (Sigma-Aldrich, Taufkirchen, Germany), were treated with proteinase K (Sigma-Aldrich, Taufkirchen, Germany) $(100 \mu \mathrm{g} / \mathrm{mL}, 30 \mathrm{~min})$. After deactivation with PMSF (phenylmethylsulfonyl fluoride) (Roche, Mannheim, Germany) and AEBSF (4-(2- aminoethyl) benzenesulfonyl fluoride hydrocholoride) (Sigma-Aldrich, Taufkirchen, Germany), the samples were analyzed by immunoblot as described above. $\beta$-lactamase activity in OMVs was quantified with the $\beta$-lactamase activity assay kit (Sigma-Aldrich, Taufkirchen, Germany) as recommended by the manufacturer.

\subsection{GAS Protection against Amoxicillin via $\beta$-Lactamase-Carrying, Amoxicillin-Induced NTHi OMVs}

To determine GAS protection against amoxicillin via $\beta$-lactamase-carrying, amoxicillininduced NTHi OMVs (hereafter designated NTHi OMVs ${ }_{\beta L+A M X+}$ ), GAS was cultured for $24 \mathrm{~h}$ in BHI broth supplemented with amoxicillin in concentrations reported in the tonsils of amoxicillin-treated patients $(0.17 \mu \mathrm{g} / \mathrm{mL}$ or $3.9 \mu \mathrm{g} / \mathrm{mL})$ [30,32], and: (i) NTHi $\mathrm{OMVs}_{\beta \mathrm{L}+\mathrm{AMX}}\left(724 \mu \mathrm{g} / \mathrm{mL}\right.$ or $1.2 \mathrm{mg} / \mathrm{mL}$ of OMV protein); or (ii) NTHi OMVs ${ }_{\beta \mathrm{L}+\mathrm{AMX}+}$ $(1.2 \mathrm{mg} / \mathrm{mL}$ of OMV protein) and potassium clavulanate (Sigma-Aldrich, Taufkirchen, Germany; $25 \mu \mathrm{g} / \mathrm{mL}$ ); or (iii) $\beta$-lactamase-negative NTHi OMVs $(1.2 \mathrm{mg} / \mathrm{mL}$ of OMV protein) from a control amoxicillin-susceptible NTHi from our collection (amoxicillin minimal inhibitory concentration (MIC) of $0.5 \mu \mathrm{g} / \mathrm{mL}$ ). Growth was quantified by determination of colony-forming units (CFU)/mL. The amounts of NTHi OMVs ${ }_{\beta L+A M X}$ used, i.e., $724 \mu \mathrm{g} / \mathrm{mL}$ and $1.2 \mathrm{mg} / \mathrm{mL}$, corresponded to those produced by NTHi in the presence of amoxicillin concentrations of $0.17 \mu \mathrm{g} / \mathrm{mL}$ and $3.9 \mu \mathrm{g} / \mathrm{mL}$, respectively (Table 1). GAS culture in BHI broth without amoxicillin and in BHI broth with amoxicillin $(0.17 \mu \mathrm{g} / \mathrm{mL}$ or $3.9 \mu \mathrm{g} / \mathrm{mL}$ ) but without NTHi OMVs ${ }_{\beta \mathrm{L}+\mathrm{AMX}}$ served as a control of GAS growth and of amoxicillin-mediated GAS inhibition, respectively. After $24 \mathrm{~h}$, each culture was streaked on BHI agar without amoxicillin and with amoxicillin $(0.17 \mu \mathrm{g} / \mathrm{mL}$ or $3.9 \mu \mathrm{g} / \mathrm{mL})$ and checked for growth after overnight incubation.

Table 1. Effect of amoxicillin on protein concentrations of OMVs produced by NTHi patient's isolate.

\begin{tabular}{lc}
\hline NTHi OMVs Produced under Conditions & OMV Protein Concentration $(\mu \mathrm{g} / \mathrm{mL})$ \\
\hline BHI broth without amoxicillin & 325 \\
BHI broth + amoxicillin $0.17 \mu \mathrm{g} / \mathrm{mL}^{1}$ & 724 \\
BHI broth + amoxicillin $1.1 \mu \mathrm{g} / \mathrm{mL}^{2}$ & 989 \\
BHI broth + amoxicillin $3.9 \mu \mathrm{g} / \mathrm{mL}^{3}$ & 1200 \\
\hline
\end{tabular}

NTHi, nontypeable Haemophilus influenzae; OMVs, outer membrane vesicles; BHI, brain heart infusion. ${ }^{1,2,3}$ Amoxicillin concentrations reported in the tonsils of amoxicillin-treated patients who underwent tonsillectomy [30-32].

\subsection{The Influence of NTHi OMVs $s_{\beta L+A M X+}$ on Amoxicillin MIC for GAS}

To determine the influence of NTHi OMVs $\beta_{\beta}+\mathrm{AMX}+$ on amoxicillin MIC for GAS, MIC was determined for: (i) GAS alone; (ii) GAS supplemented with NTHi OMVs $s_{\beta L+A M X+}$ (724 $\mathrm{\mu g} / \mathrm{mL}$ or $1.2 \mathrm{mg} / \mathrm{mL}$ of OMV protein); (iii) GAS supplemented with NTHi OMVs $\mathrm{s}_{\beta \mathrm{L}+\mathrm{AMX}+}$ (1.2 $\mathrm{mg} / \mathrm{mL}$ of OMV protein) and potassium clavulanate $(25 \mu \mathrm{g} / \mathrm{mL})$; and iv) GAS supplemented with $\beta$-lactamase-negative OMVs from the control amoxicillin-susceptible NTHi $(1.2 \mathrm{mg} / \mathrm{mL}$ of OMV protein).

\subsection{In Vivo Detection of OMV-Producing H. Influenzae and $\beta$-Lactamase-Carrying OMVs}

Pharyngotonsillar swabs, exudate from tonsillar crypts, and saliva were taken from the patient and processed for transmission electron microscopy as described previously [34] with minor modifications. Briefly, the pharyngotonsillar swabs and swabs soaked with crypt exudate were placed into phosphate-buffered saline (PBS) with $2 \%$ paraformaldehyde and $0.2 \%$ glutaraldehyde (Sigma-Aldrich, Taufkirchen, Germany), vigorously vortexed to release bacteria and OMVs, fixed for $30 \mathrm{~min}$, ultracentrifuged $(52,000 \times \mathrm{g}, 30 \mathrm{~min})$, 
and the pellets were embedded in 6\% gelatine (fish skin gelatine, BioTrend, Miramar Beach, USA). Saliva (15 mL) was mixed with $15 \mathrm{~mL}$ of PBS, and vesicles were collected by ultracentrifugation and embedded in gelatine as above. Ultrathin ( $50 \mathrm{~nm})$ cryosections were cut from the gelatine blocks (cryo-ultramicrotome Leica Ultracut EM UC7; Leica Microsystem, Wetzlar, Germany), placed on a formvar film and carbon-coated copper grid (Sigma-Aldrich, Taufkirchen, Germany), and stained with a mouse monoclonal anti- $\beta$ lactamase antibody (Abcam, Cambridge, UK, Cat\# ab12251, RRID:AB_298974) and goat anti-mouse IgG conjugated with $10 \mathrm{~nm}$ gold (Abcam, Cambridge, UK, Cat\# ab39619, RRID:AB_954440). The preparations were postfixed with glutaraldehyde (Sigma-Aldrich, Taufkirchen, Germany), contrasted with uranyl acetate (Thermo Fisher Scientific, Erlangen, Germany), and examined with an HT7800 transmission electron microscope (Hitachi High-Tech Analytical Science, Prague, Czech Republic).

\subsection{Statistical Analysis}

Data were analyzed with one-way ANOVA (analysis of variance); $p<0.05$ was considered significant.

\section{Results}

\subsection{Amoxicillin Treatment Failure in a Patient with GAS Pharyngotonsillitis}

The throat culture of the patient with pharyngotonsillitis grew GAS, which was susceptible to penicillin, ampicillin, and amoxicillin (Table 2). The patient was treated with amoxicillin (750 mg three times daily, p.o.) for 10 days, but no improvement was observed. A repeated throat culture performed after completing the amoxicillin treatment continued growing amoxicillin-susceptible GAS. Moreover, this second culture revealed NTHi, which was resistant to amoxicillin (MIC of $16 \mu \mathrm{g} / \mathrm{mL}$ ), carried the bla $a_{\mathrm{TEM}-1}$ gene, and was likely selected by the amoxicillin therapy. It was susceptible to amoxicillin/clavulanate (Table 2). The patient was treated with amoxicillin/clavulanate ( $1 \mathrm{~g}$ twice daily, p.o.) and fully recovered after 4 days; the therapy was continued for up to 10 days. Control throat cultures performed 2 days and 10 days after termination of the amoxicillin/clavulanate therapy were negative for GAS and NTHi.

Table 2. Antimicrobial susceptibilities of GAS and NTHi isolated from the patient's throat culture.

\begin{tabular}{|c|c|c|c|c|c|}
\hline \multirow{2}{*}{ Isolate } & \multicolumn{2}{|c|}{ Disc Diffusion Method } & \multicolumn{2}{|c|}{$\begin{array}{l}\text { Broth Microdilution Method } \\
\text { MIC }(\mu \mathrm{g} / \mathrm{mL})\end{array}$} & \multirow{2}{*}{ PCR $b l a_{\text {TEM-1 }}$} \\
\hline & Susceptible to & Resistant to & Amoxicillin & $\begin{array}{l}\text { Amoxicillin/ } \\
\text { Clavulanate }\end{array}$ & \\
\hline GAS $^{1}$ & \multirow{2}{*}{$\begin{array}{c}\text { Penicillin, Ampicillin Amoxicillin } \\
\text { Amoxicillin/ } \\
\text { clavulanate }\end{array}$} & \multirow{2}{*}{$\begin{array}{l}\text { None of tested } \\
\text { Ampicillin } \\
\text { Amoxicillin }\end{array}$} & 0.016 & 0.016 & Negative \\
\hline NTHi & & & 16 & 0.5 & Positive \\
\hline
\end{tabular}

GAS, Streptococcus pyogenes group A; NTHi, nontypeable Haemophilus influenzae. ${ }^{1}$ The data refer to the GAS isolates obtained before amoxicillin treatment and after amoxicillin treatment.

\subsection{NTHi Patient's Isolate Secretes $\beta$-Lactamase-Carrying OMVs That Are Induced by Amoxicillin}

To gain insight into the role of NTHi OMVs in the amoxicillin therapy failure, we isolated OMVs from the NTHi patient's isolate (Figure 1a) and analyzed them for the presence of $\beta$-lactamase and $\beta$-lactamase activity. The OMVs contained $\beta$-lactamase (Figure $1 b$ ), which was located inside OMVs, as demonstrated by its protection against proteinase $\mathrm{K}$ (PK) in the PK assay (Figure 1b). The $\beta$-lactamase was enzymatically active, as evidenced by the ability of OMVs to cleave the $\beta$-lactamase substrate nitrocefin (Figure 1c). Notably, the $\beta$-lactamase activity (Figure 1c) and the amount (Figure 1d) of OMVs produced by NTHi at the time of its isolation from the patient's tonsils (reflecting the situation in vivo during therapy) significantly decreased when the isolate was passaged in vitro in a medium without amoxicillin, and significantly increased when amoxicillin in concentrations reported in 
the tonsillar tissue during amoxicillin treatment $(0.17 \mu \mathrm{g} / \mathrm{mL}$ to $3.9 \mu \mathrm{g} / \mathrm{mL})$ [30-32] was added to the NTHi culture (Figure 1c,d). Thus, amoxicillin in concentrations reached in the tonsils during therapy significantly increased secretion and enzymatic activity of NTHi $\beta$ lactamase-containing OMVs. This led us to hypothesize that these $\beta$-lactamase-containing, amoxicillin-induced OMVs (hereafter termed NTHi OMVs $\mathrm{SL}_{\mathrm{L}+\mathrm{AMX}+}$ ) were involved in the amoxicillin failure to eradicate GAS from the patient by protecting GAS against amoxicillin.

(a)

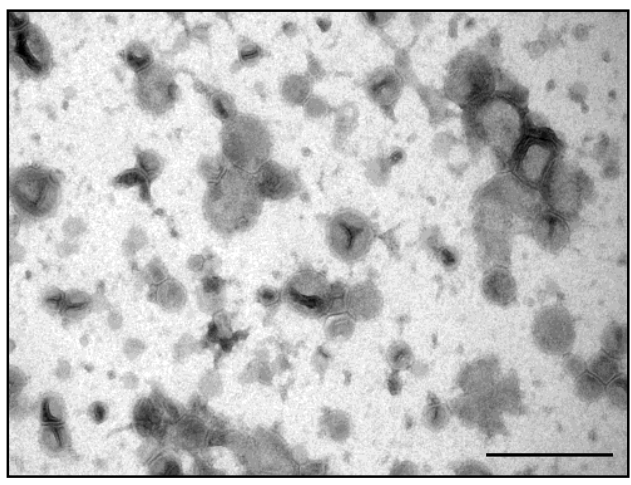

(c)

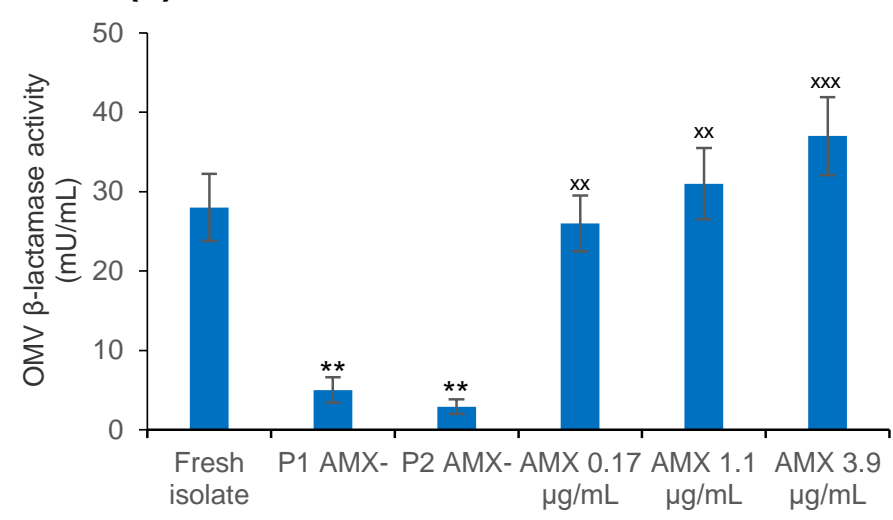

(b)

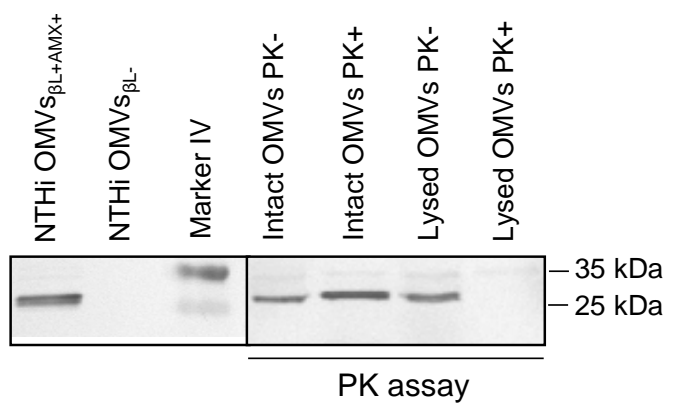

(d)

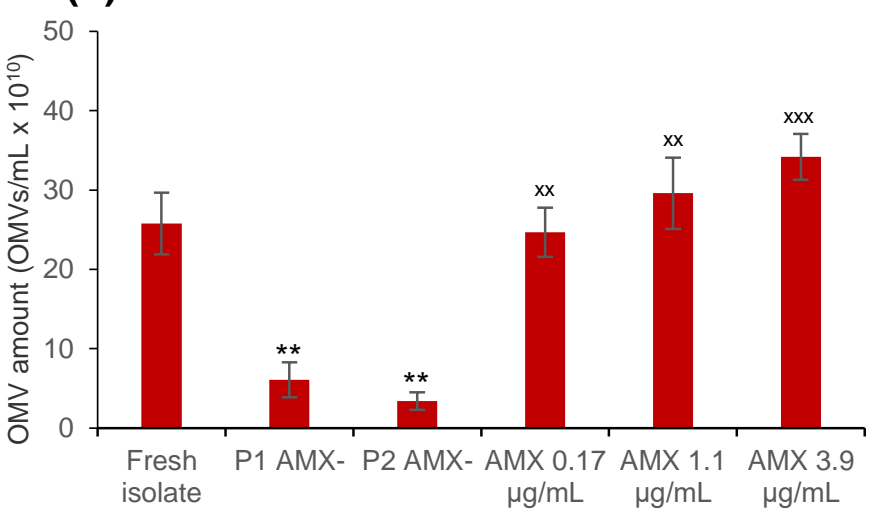

Figure 1. Nontypeable Haemophilus influenzae (NTHi) isolated from the patient's throat culture secretes $\beta$-lactamase-carrying OMVs, which are induced by amoxicillin (NTHi OMVs $\mathrm{SL}_{+} \mathrm{AMX}+$ ): (a) electron microscopy of negatively stained NTHi $\mathrm{OMVs}_{\beta \mathrm{L}+\mathrm{AMX}+}$; scale bar, $200 \mathrm{~nm}$. (b) OMV immunoblot with anti- $\beta$-lactamase antibody. Left panel: lane 1, NTHi $\mathrm{OMVs}_{\beta \mathrm{L}+\mathrm{AMX}+}$, lane 2, control $\beta$-lactamase-negative OMVs (NTHi OMVs $\mathrm{SL}_{-}$), lane 3, protein size marker (the marker band sizes are on the right side); $\beta$-lactamase is $\sim 32 \mathrm{kDa}$. Right panel: OMVs $\mathrm{sL}_{\beta+\mathrm{AMX}}$ subjected to proteinase $\mathrm{K}$ (PK) assay, which demonstrates intravesicular localization of $\beta$-lactamase. (c,d) $\beta$-lactamase activities (c) and the amounts (d) of OMVs produced by NTHi freshly isolated from the patient's throat culture (fresh isolate), by NTHi passaged twice in BHI broth without amoxicillin (P1 and P2, AMX-), and by NTHi from passage 2 grown in BHI broth with amoxicillin concentrations reported in the tonsils during therapy $(0.17 \mu \mathrm{g} / \mathrm{mL}, 1.1 \mu \mathrm{g} / \mathrm{mL}$, or $3.9 \mu \mathrm{g} / \mathrm{mL})$. Data are presented as means \pm standard deviations from three independent experiments; ${ }^{* *} p<0.01$ compared to fresh isolate; ${ }^{\mathrm{xx}} p<0.01$ compared to P2 AMX-; ${ }^{\mathrm{xx}} p<0.001$ compared to P2 AMX- (statistical analysis was performed with one-way ANOVA).

\subsection{NTHi OMVs ${ }_{\beta L+A M X+}$ Protect GAS against Bactericidal Concentrations of Amoxicillin}

To test the hypothesis that NTHi OMVs ${ }_{\beta L}+A M X+$ are involved in the amoxicillin failure to eradicate GAS from the patient, we determined whether these OMVs protect GAS against the reported amoxicillin tonsillar concentrations of $0.17 \mu \mathrm{g} / \mathrm{mL}$ and $3.9 \mu \mathrm{g} / \mathrm{mL}$ [30,32], which represent $~ 10$-fold and 244-fold MICs, respectively, for this isolate (MIC of $0.016 \mu \mathrm{g} / \mathrm{mL}$ ) (Table 2). To this end, GAS growth was monitored for $24 \mathrm{~h}$ in the presence of each amoxicillin concentration and NTHi OMVs $\mathrm{SL}_{+\mathrm{AMX}+}$ in the doses of $724 \mu \mathrm{g} / \mathrm{mL}$ or $1.2 \mathrm{mg} / \mathrm{mL}$, which were induced by the respective amoxicillin concentrations (Table 1). Indeed, each NTHi OMVs ${ }_{\beta L+A M X+}$ dose protected GAS against amoxicillin, with 
the protection being slightly delayed with $724 \mu \mathrm{g} / \mathrm{mL}$ of NTHi OMVs $\mathrm{s}_{\beta+\mathrm{AMX}}$ against $3.9 \mu \mathrm{g} / \mathrm{mL}$ of amoxicillin (Figure $2 \mathrm{a}, \mathrm{b}$ ). This demonstrated that NTHi OMVs $\mathrm{s}_{\beta+\mathrm{AMX}+}$ in the amounts induced by amoxicillin concentrations reached in the tonsils during therapy protected GAS against bactericidal effect of amoxicillin. No GAS protection was conferred by $\beta$-lactamase-negative OMVs from a control amoxicillin-susceptible NTHi (Figure $2 \mathrm{a}, \mathrm{b}$ ), indicating that the $\beta$-lactamase associated with NTHi OMVs ${ }_{\beta \mathrm{L}+\mathrm{AMX}}+$ was the GAS-protecting component.
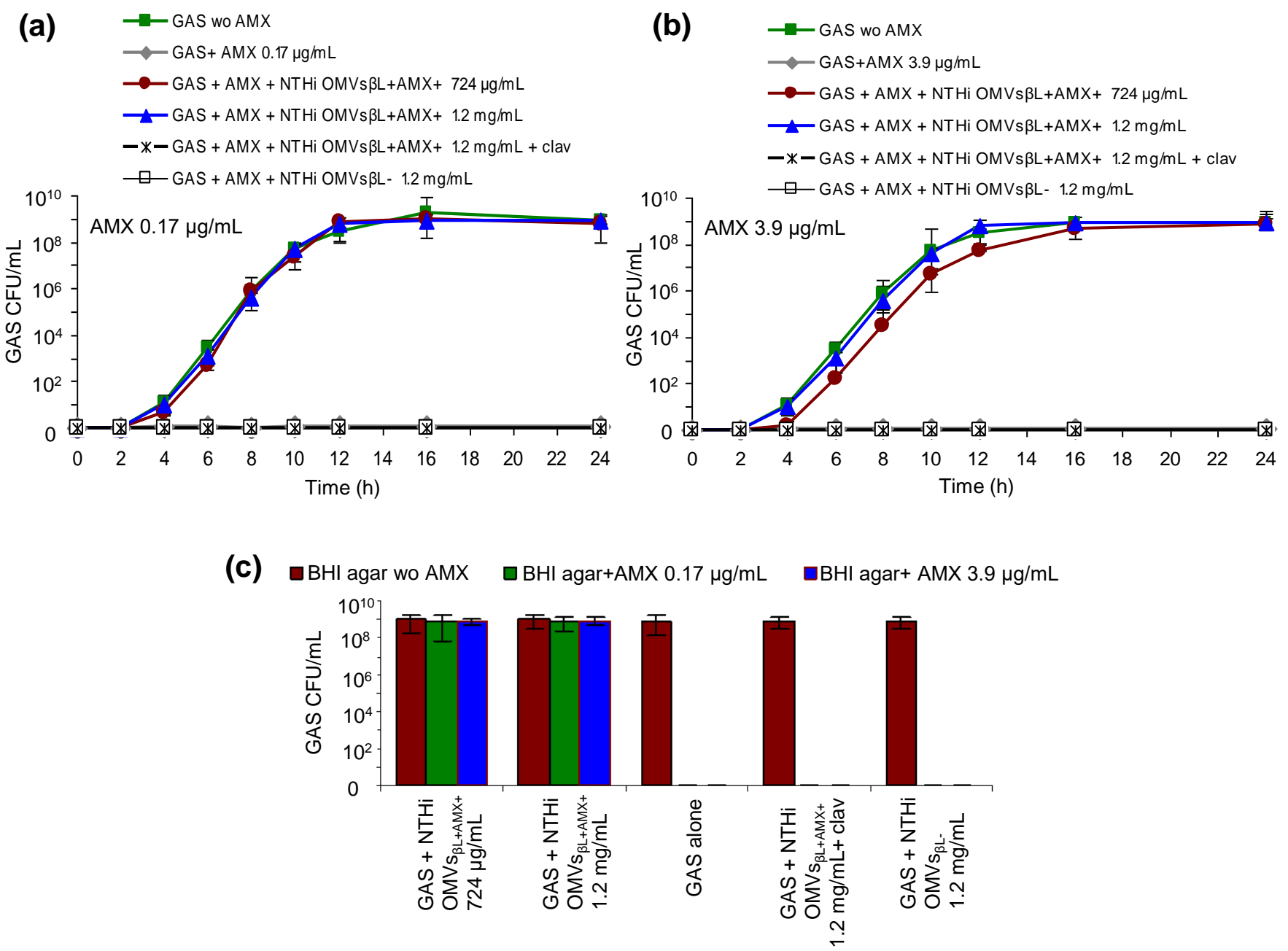

Figure 2. Amoxicillin-induced, $\beta$-lactamase-carrying OMVs from amoxicillin-resistant nontypeable Haemophilus influenzae $\left(\mathrm{NTHi} \mathrm{OMVs}_{\beta \mathrm{L}+\mathrm{AMX}}\right)$ protect GAS against bactericidal concentrations of amoxicillin: $(\mathbf{a}, \mathbf{b})$ GAS growth in BHI broth without amoxicillin or with amoxicillin $0.17 \mu \mathrm{g} / \mathrm{mL}(\mathbf{a})$ or $3.9 \mu \mathrm{g} / \mathrm{mL}(\mathbf{b})$ in the absence or presence of the indicated amounts of NTHi OMVs $\beta_{\mathrm{L}+\mathrm{AMX}+}$ without or with clavulanate, or in the presence of control $\beta$-lactamase-negative NTHi OMVs $\left(\mathrm{NTHi}_{\mathrm{OMVs}} \mathrm{L}_{-}\right)$. Data are means \pm standard deviations (SDs) from three independent experiments. (c) Growth of GAS from $24 \mathrm{~h}$ cultures shown in $(\mathbf{a}, \mathbf{b})$ on BHI agar without or with amoxicillin. Data are means \pm SDs from three independent experiments.

\subsection{NTHi OMVs $s_{\beta L+A M X+}$ Increase Amoxicillin MIC for GAS}

To elucidate the basis for the NTHi OMVs $s_{\beta L+A M X+}$-mediated GAS protection against amoxicillin, we determined the effect of these OMVs on amoxicillin MIC for GAS. We found that in the presence of $724 \mu \mathrm{g} / \mathrm{mL}$ and $1.2 \mathrm{mg} / \mathrm{mL}$ of NTHi OMVs $\mathrm{BL}_{+} \mathrm{AMX}_{+}$, the amoxicillin MIC for GAS increased from $0.016 \mu \mathrm{g} / \mathrm{mL}$ to $4 \mu \mathrm{g} / \mathrm{mL}$ (250-fold) and to $16 \mu \mathrm{g} / \mathrm{mL}$ (1000-fold), respectively (Table 3), making GAS resistant to amoxicillin. Importantly, both the amoxicillin MIC increase for GAS and the GAS protection against amoxicillin via NTHi OMVs $s_{\beta}+A M X+$ were inhibited by the $\beta$-lactamase inhibitor clavu- 
lanate (Figure 2a,b, Table 3), confirming that these effects were mediated by the NTHi $\mathrm{OMVs}_{\mathrm{BL}+\mathrm{AMX}+}$-associated $\beta$-lactamase. Moreover, GAS failed to grow on a medium with amoxicillin in the absence of NTHi OMVs $\beta \mathrm{L}_{+} \mathrm{AMX}+$ (Figure 2c), demonstrating that these OMVs, not acquisition of the bla $a_{\mathrm{TEM}-1}$ gene from NTHi, accounted for its amoxicillin resistance. The absence of $b l a_{\text {TEM-1 }}$ in GAS was confirmed by PCR (Table 2).

Table 3. The influence of NTHi OMVs $s_{\beta+A M X+}$ on amoxicillin MIC for GAS isolated from the patient's throat culture.

\begin{tabular}{lc}
\hline GAS Culture Tested for Amoxicillin MIC & Amoxicillin MIC for GAS $(\mu \mathrm{g} / \mathrm{mL})$ \\
\hline GAS alone & 0.016 \\
GAS + NTHi OMVs $_{\beta L+A M X+}{ }^{1}(724 \mu \mathrm{g} / \mathrm{mL})$ & 4 \\
GAS + NTHi OMVs & 16 \\
GAS + NTHi OMV $+(1.2 \mathrm{mg} / \mathrm{mL})$ & 0.032 \\
GAS + NTHi OMVs $_{\beta \mathrm{L}-\mathrm{AMX}-}{ }^{2}(1.2 \mathrm{mg} / \mathrm{mL})$ & 0.016 \\
\hline
\end{tabular}

GAS, Streptococcus pyogenes group A; MIC, minimal inhibitory concentration; NTHi, nontypeable Haemophilus influenzae. ${ }^{1} \mathrm{NTHi} O M \mathrm{Ss}_{\beta \mathrm{L}+\mathrm{AMX}+}, \beta$-lactamase-carrying, amoxicillin-induced OMVs from NTHi patient's isolate.

2 NTHi OMVs ${ }_{\beta L-}, \beta$-lactamase-negative OMVs from a control amoxicillin-susceptible NTHi.

\subsection{NTHi $\beta$-Lactamase-Carrying OMVs Are Secreted In Vivo at the Infection Site}

To provide a final piece of evidence for the involvement of NTHi OMVs $s_{\beta L+A M X}+$ in the amoxicillin therapy failure, we searched for their secretion in vivo at the site of infection. We identified NTHi bacteria secreting $\beta$-lactamase-carrying OMVs as well as released, free $\beta$-lactamase-carrying OMVs in the patient's pharyngotonsillar swabs (Figure $3 a)$, tonsillar crypt exudate (Figure 3b), and saliva (Figure 3c). Taken together, our findings demonstrate that amoxicillin-resistant NTHi colonizing the pharyngotonsillar mucosa of the GAS-infected patient secreted in situ $\beta$-lactamase-carrying OMVs, which were inducible by amoxicillin and protected GAS against the antibiotic, thereby accounting for the therapy failure.

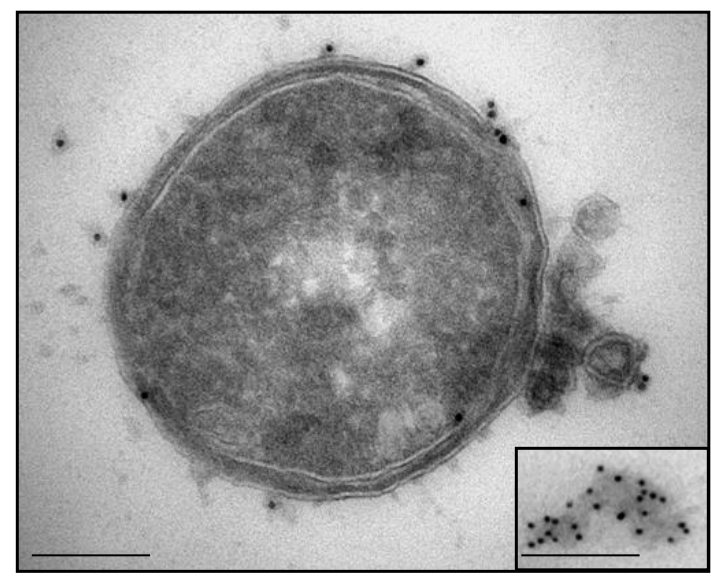

(a)

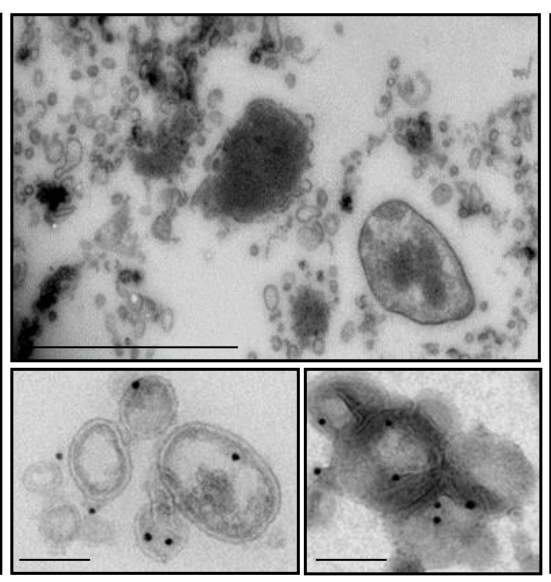

(b)

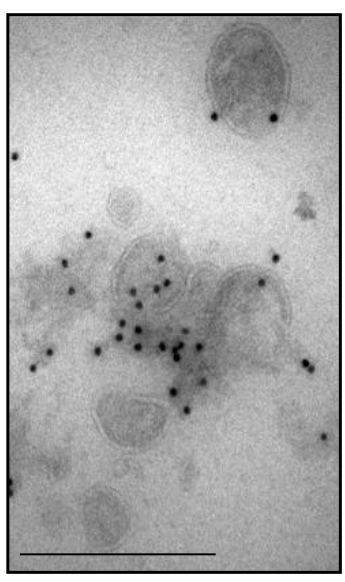

(c)

Figure 3. Transmission electron microscopy of the patient's pharyngotonsillar swab, crypt exudate, and saliva demonstrating in vivo secretion of $\beta$-lactamase-carrying NTHi OMVs: (a) NTHi secreting $\beta$-lactamase-carrying OMVs and released $\beta$ lactamase-carrying OMVs (inset) in pharyngotonsillar swab. (b) NTHi bacteria surrounded by large amounts of OMVs (top) and released $\beta$-lactamase-carrying OMVs (bottom) in tonsillar crypt exudate. (c) $\beta$-lactamase-carrying OMVs in saliva. Pictures are ultrathin cryosections stained (except for $(\mathbf{b})$ top which is unstained) with anti- $\beta$-lactamase antibody and $10 \mathrm{~nm}$ gold-conjugated secondary antibody. Scale bars: (a,c), $200 \mathrm{~nm}$; (b) top, $1 \mu \mathrm{m}$, bottom, $100 \mathrm{~nm}$. 


\section{Discussion}

This study brings a new insight into the mechanisms of amoxicillin therapy failure in patients with GAS pharyngotonsillitis. The involvement of $\beta$-lactamase-carrying OMVs secreted by NTHi colonizing the patient's pharynx and tonsils in this failure is supported: (i) by the induction of NTHi OMV secretion and OMV-associated $\beta$-lactamase activity by amoxicillin concentrations reached in the tonsils during therapy; (ii) by the ability of NTHi OMVs ${ }_{\beta L+A M X+}$ to significantly increase amoxicillin MIC for GAS and to protect GAS against bactericidal concentrations of amoxicillin; (iii) by the inhibition of each of these NTHi OMVs ${ }_{\beta L+A M X+}-$ mediated effects by the $\beta$-lactamase inhibitor clavulanate; (iv) by the inability of GAS to resist amoxicillin in the absence of NTHi OMVs $\beta_{\beta L+A M X+}$, which is in accordance with its excellent susceptibility to amoxicillin [1]; and (v) by the secretion of NTHi OMVs $s_{\beta L+A M X+}$ in vivo at the site of infection. This is, to the best of our knowledge, the first evidence that OMV-mediated protection of bacteria against $\beta$-lactam antibiotics previously observed under in vitro conditions $[18,22-26]$ has a clinical parallel in the ability of $\beta$-lactamase-carrying OMVs secreted in vivo to protect pathogens against $\beta$-lactams during patients' treatment, thus leading to therapy failure. For patients with GAS pharyngotonsillitis, this mechanism may be of a particular importance, since more than one fourth of children with GAS pharyngotonsillitis have their tonsils colonized with H. influenzae or M. catarrhalis [37], both of which can produce $\beta$-lactamase-carrying OMVs $([22,23]$, this study). Mechanistically, the structural identity between the bacterial outer membrane and OMV membrane allows $\beta$-lactams to enter, through the porin channels, the OMV lumen, where $\beta$-lactamase, originating from the bacterial periplasm, is located and hydrolyzes the antibiotics [22-24,38]. Through this process, OMVs secreted outside bacterial cells serve as a first line of protection against $\beta$-lactams before the antibiotics reach the target bacterial population. Since OMVs secreted by Gram-negative $\beta$-lactam-resistant bacteria in vitro carry a broad spectrum of $\beta$-lactamases [18,22-26], it is likely that a similar mechanism that we have described for amoxicillin plays a role in therapeutic failures of other $\beta$-lactam antibiotics including carbapenems, which are the "last resort" $\beta$-lactams used to combat multidrug resistant pathogens [26]. Moreover, experimental data suggest that OMVs may also be involved in antimicrobial resistance in other ways, including the sequestration of membrane active antibiotics (polymyxin B, colistin) $[8,20,21,39]$ and dissemination of antibiotic resistance genes $[33,40,41]$. Thus, OMVs may serve as universal bacterial tools contributing, by different mechanisms, to antibiotic resistance. A broad involvement of OMVs in antimicrobial resistance is strongly supported by the observations that the increase in OMV secretion (e.g., by hypervesiculating mutans) increased the resistance, and the reduction in or inhibition of OMV secretion increased the susceptibility of various bacteria to a range of antibiotics $[21,39,42]$.

The mechanism by which amoxicillin (and other $\beta$-lactam antibiotics such as imipenem [17] and meropenem [16]) increases OMV production is presently not known. Based on the models of OMV biogenesis [6] and the mechanism of action of $\beta$-lactam antibiotics [43], we hypothesize that the inhibition of peptidoglycan polymerization due to the $\beta$-lactam binding to the penicillin-binding proteins [43] plays a key role. This hypothesis is supported by the occurrence of OMV budding at the sites of locally decreased crosslinking between the peptidoglycan and the outer membrane [6]; it is in accordance with the peptidoglycan being a central structure that accounts, via its crosslinks with various membrane proteins, for the stability of the bacterial envelope [6].

The therapeutic success of amoxicillin/clavulanate in our patient demonstrated that amoxicillin's failure to eradicate GAS was not due to a poor tonsillar penetration of the drug. However, the amoxicillin concentration in the patient's tonsils could not be determined, as he did not undergo a tonsillectomy. This is the reason why we used the concentrations reported in the tonsils of amoxicillin-treated patients who did undergo a tonsillectomy [30-32] to determine the effects of amoxicillin on the amount of OMVs produced by NTHi and the OMV $\beta$-lactamase activity. Since these concentrations encompass a broad range $(0.17 \mu \mathrm{g} / \mathrm{mL}-3.9 \mu \mathrm{g} / \mathrm{mL})$, it is likely that the amoxicillin concentration in the tonsils of our patient was within this range. To 
further evaluate the mechanism of $\beta$-lactam therapy failure reported in this study in other clinically relevant situations, we will continue our investigations in additional patients with pharyngotonsillitis, in whom a coinfection with $\beta$-lactam-susceptible (GAS and others) and beta-lactam-resistant bacteria is detected, and the $\beta$-lactam antibiotic therapy failure occurs.

\section{Conclusions}

The pathogen protection via $\beta$-lactamase-carrying OMVs secreted in situ by the flora coinhabiting the infection site represents a yet underestimated mechanism of $\beta$-lactam therapy failure. The extent of the involvement of this mechanism in $\beta$-lactam therapy failure in clinical praxis needs to be evaluated in further clinical-microbiological studies. The emerging role of OMVs in antibiotic resistance should be taken into account in strategies directed at combating this serious medical and public health problem.

Author Contributions: Conceptualization, M.B. and A.M.; methodology: M.B., O.D. and O.N.; validation, M.B.; formal analysis, M.B.; investigation, M.B., O.D. and O.N.; resources, M.B. and O.N.; writing—original draft preparation, M.B.; writing—review and editing, A.M., O.D., O.N. and M.B.; visualization, O.D. and M.B.; supervision, A.M.; project administration, M.B.; funding acquisition, M.B., O.D. and A.M. All authors have read and agreed to the published version of the manuscript.

Funding: This research was funded by the Deutsche Forschungsgemeinschaft (DFG): 194468054SFB 1009, B04, and Ministry of Health of the Czech Republic: NIPH 75010330.

Institutional Review Board Statement: The study was conducted according to the guidelines of the Declaration of Helsinki, and approved by the Ethics Committee of the National Institute of Public Health, Prague (protocol code EK-SZU/08076/2021) on 1 June 2021.

Informed Consent Statement: Informed consent was obtained from the patient involved in the study.

Data Availability Statement: The datasets supporting the results of this article are included within the article.

Acknowledgments: We thank the patient for his agreement to participate in the study and to publish the data.

Conflicts of Interest: The authors declare no conflict of interest. The funders had no roles in the design of the study; in the collection, analyses, or interpretation of data; in the writing of the manuscript, or in the decision to publish the results.

\section{References}

1. Shulman, S.T.; Bisno, A.L.; Clegg, H.W.; Gerber, M.A.; Kaplan, E.L.; Lee, G.; Martin, J.M.; Van Beneden, C. Clinical practice guideline for the diagnosis and management of group A streptococcal pharyngitis: 2012 update by the Infectious Diseases Society of America. Clin. Infect. Dis. 2012, 55, e86-e102. [CrossRef]

2. Brook, I. Overcoming penicillin failures in the treatment of Group A streptococcal pharyngo-tonsillitis. Int. J. Pediatr. Otorhinolaryngol. 2007, 71, 1501-1508. [CrossRef]

3. Ellis, T.N.; Kuehn, M.J. Virulence and immunomodulatory roles of bacterial outer membrane vesicles. Microbiol. Mol. Biol. Rev. 2010, 74, 81-94. [CrossRef]

4. Toyofuku, M.; Nomura, N.; Eberl, L. Types and origins of bacterial membrane vesicles. Nat. Rev. Microbiol. 2019, 17, 13-24. [CrossRef] [PubMed]

5. Guerrero-Mandujano, A.; Hernández-Cortez, C.; Ibarra, J.A.; Castro-Escarpulli, G. The outer membrane vesicles: Secretion system type zero. Traffic 2017, 18, 425-432. [CrossRef] [PubMed]

6. Schwechheimer, C.; Kuehn, M.J. Outer-membrane vesicles from Gram-negative bacteria: Biogenesis and functions. Nat. Rev. Microbiol. 2015, 13, 605-619. [CrossRef] [PubMed]

7. Roier, S.; Zingl, F.G.; Cakar, F.; Durakovic, S.; Kohl, P.; Eichmann, T.O.; Klug, L.; Gadermaier, B.; Weinzerl, K.; Prassl, R.; et al. A novel mechanism for the biogenesis of outer membrane vesicles in Gram-negative bacteria. Nat. Commun. 2016, 7, 10515. [CrossRef]

8. MacDonald, I.A.; Kuehn, M.J. Offense and defense: Microbial membrane vesicles play both ways. Res. Microbiol. 2012, 163, 607-618. [CrossRef]

9. Caruana, J.C.; Walper, S.A. Bacterial membrane vesicles as mediators of microbe-Microbe and microbe-Host community interactions. Front. Microbiol. 2020, 11, 432. [CrossRef]

10. Rueter, C.; Bielaszewska, M. Secretion and delivery of intestinal pathogenic Escherichia coli virulence factors via outer membrane vesicles. Front. Cell. Infect. Microbiol. 2020, 10, 91. [CrossRef] 
11. Tan, T.T.; Morgelin, M.; Forsgren, A.; Riesbeck, K. Haemophilus influenzae survival during complement-mediated attacks is promoted by Moraxella catarrhalis outer membrane vesicles. J. Infect. Dis. 2007, 195, 1661-1670. [CrossRef]

12. Namork, E.; Brandtzaeg, P. Fatal meningococcal septicaemia with “blebbing" meningococcus. Lancet 2002, 360, 1741. [CrossRef]

13. McBroom, A.J.; Kuehn, M.J. Release of outer membrane vesicles by Gram-negative bacteria is a novel envelope stress response. Mol. Microbiol. 2007, 63, 545-558. [CrossRef] [PubMed]

14. Orench-Rivera, N.; Kuehn, M.J. Environmentally controlled bacterial vesicle-mediated export. Cell. Microbiol. 2016, 18, 1525-1536. [CrossRef] [PubMed]

15. Bauwens, A.; Kunsmann, L.; Marejková, M.; Zhang, W.; Karch, H.; Bielaszewska, M.; Mellmann, A. Intrahost milieu modulates production of outer membrane vesicles, vesicle-associated Shiga toxin 2a and cytotoxicity in Escherichia coli O157:H7 and O104:H4 Environ. Microbiol. Rep. 2017, 9, 626-634. [CrossRef] [PubMed]

16. Bauwens, A.; Kunsmann, L.; Karch, H.; Mellmann, A.; Bielaszewska, M. Antibiotic-mediated modulations of outer membrane vesicles in enterohemorrhagic Escherichia coli O104:H4 and O157:H7. Antimicrob. Agents Chemother. 2017, 61, e00937-17. [CrossRef] [PubMed]

17. Devos, S.; Van Oudenhove, L.; Stremersch, S.; Van Putte, W.; De Rycke, R.; Van Driessche, G.; Vitse, J.; Raemdonck, K.; Devreese, B. The effect of imipenem and diffusible signaling factors on the secretion of outer membrane vesicles and associated Ax21 proteins in Stenotrophomonas maltophilia. Front. Microbiol. 2015, 6, 298. [CrossRef] [PubMed]

18. Devos, S.; Stremersch, S.; Raemdonck, K.; Braeckmans, K.; Devreese, B. Intra- and interspecies effects of outer membrane vesicles from Stenotrophomonas maltophilia on $\beta$-lactam resistance. Antimicrob. Agents Chemother. 2016, 60, 2516-2518. [CrossRef] [PubMed]

19. Maredia, R.; Devineni, N.; Lentz, P.; Dallo, S.F.; Yu, J.; Guentzel, N.; Chambers, J.; Arulanandam, B.; Haskins, W.E.; Weitao, T. Vesiculation from Pseudomonas aeruginosa under SOS. Sci. World J. 2012, 2012, 402919. [CrossRef] [PubMed]

20. Kulkarni, H.M.; Nagaraj, R.; Jagannadham, M.V. Protective role of E. coli outer membrane vesicles against antibiotics. Microbiol. Res. 2015, 181, 1-7. [CrossRef]

21. Manning, A.J.; Kuehn, M.J. Contribution of bacterial outer membrane vesicles to innate bacterial defense. BMC Microbiol. 2011, 11, 258. [CrossRef]

22. Schaar, V.; Nordström, T.; Mörgelin, M.; Riesbeck, K. Moraxella catarrhalis outer membrane vesicles carry $\beta$-lactamase and promote survival of Streptococcus pneumoniae and Haemophilus influenzae by inactivating amoxicillin. Antimicrob. Agents Chemother. 2011, 55, 3845-3853. [CrossRef]

23. Schaar, V.; Uddbäck, I.; Nordström, T.; Riesbeck, K. Group A streptococci are protected from amoxicillin-mediated killing by vesicles containing $\beta$-lactamase derived from Haemophilus influenzae. J. Antimicrob. Chemother. 2013, 69, 117-120. [CrossRef]

24. Kim, S.W.; Park, S.B.; Im, S.P.; Lee, J.S.; Jung, J.W.; Gong, T.W.; Lazarte, J.M.S.; Kim, J.; Seo, J.S.; Kim, J.H.; et al. Outer membrane vesicles from beta-lactam-resistant Escherichia coli enable the survival of beta-lactam-susceptible E. coli in the presence of beta-lactam antibiotics. Sci. Rep. 2018, 8, 5402. [CrossRef]

25. Stentz, R.; Horn, N.; Cross, K.; Salt, L.; Brearley, C.; Livermore, D.M.; Carding, S.R. Cephalosporinases associated with outer membrane vesicles released by Bacteroides spp. protect gut pathogens and commensals against beta-lactam antibiotics. J. Antimicrob. Chemother. 2015, 70, 701-709. [CrossRef]

26. González, L.J.; Bahr, G.; Nakashige, T.G.; Nolan, E.M.; Bonomo, R.A.; Vila, A.J. Membrane anchoring stabilizes and favors secretion of New Delhi metallo-beta-lactamase. Nat. Chem. Biol. 2016, 12, 516-522. [CrossRef] [PubMed]

27. Jorgensen, J.H.; Pfaller, M.A.; Carroll, K.C.; Landry, M.L.; Funke, G.; Richter, S.S.; Warnock, D.W. Manual of Clinical Microbiology, 11th ed.; ASM Press: Washinghton, DC, USA, 2015; pp. 383-402, 667-684.

28. Clinical and Laboratory Standards Institute (CLSI). Performance Standards for Antimicrobial Susceptibility Testing, 25th ed.; M100; CLSI: Wayne, PA, USA, 2015.

29. Scriver, S.R.; Walmsley, S.L.; Kau, C.L.; Hoban, D.J.; Brunton, J.; McGeer, A.; Moore, T.C.; Witwicki, E.; Canadian Haemophilus Study Group; Low, D.E. Determination of antimicrobial susceptibilities of Canadian isolates of Haemophilus influenzae and characterization of their beta-lactamases. Antimicrob. Agents Chemother. 1994, 38, 1678-1680. [CrossRef] [PubMed]

30. Blenk, H.; Simm, K.; Blenk, B.; Jahneke, G. Concentrations of erythromycin and amoxicillin in tonsil and sinus tissues of patients with tonsillitis and sinusitis. A comparison. Infection 1982, 10 (Suppl. 2), S108-S112. [CrossRef]

31. Averono, G.; Vidali, M.; Olina, M.; Basile, M.; Bagnati, M.; Bellomo, G.; Aluffi, P. Evaluation of amoxicillin plasma and tissue levels in pediatric patients undergoing tonsillectomy. Int. J. Pediatr. Otorhinolaryngol. 2010, 74, 995-998. [CrossRef] [PubMed]

32. Chomarat, M.; Panteix, G.; Guillaumond, B.; Dubreuil, C. Tonsillar diffusion kinetics of amoxycillin after oral administration of 1 g to adults. Eur. J. Drug Metab. Pharmacokinet. 1997, 22, 141-144. [CrossRef]

33. Bielaszewska, M.; Daniel, O.; Karch, H.; Mellmann, A. Dissemination of the bla $a_{\mathrm{CTX}-\mathrm{M}-15}$ gene among Enterobacteriaceae via outer membrane vesicles. J. Antimicrob. Chemother. 2020, 75, 2442-2451. [CrossRef]

34. Bielaszewska, M.; Greune, L.; Bauwens, A.; Dersch, P.; Mellmann, A.; Rüter, C. Virulence factor cargo and host cell interactions of Shiga toxin-producing Escherichia coli outer membrane vesicles. Methods Mol. Biol. 2021, 2291, 177-205. [CrossRef] [PubMed]

35. Willysson, A.; Stahl, A.; Karpman, D. Isolation and characterization of Shiga toxin-associated microvesicles. Methods Mol. Biol. 2021, 2291, 207-228. [CrossRef] [PubMed]

36. Kunsmann, L.; Rüter, C.; Bauwens, A.; Greune, L.; Glüder, M.; Kemper, B.; Fruth, A.; Wai, S.N.; He, X.; Lloubes, R.; et al. Virulence from vesicles: Novel mechanisms of host cell injury by Escherichia coli O104:H4 outbreak strain. Sci. Rep. 2015, 5, 13252. [CrossRef] [PubMed] 
37. Brook, I.; Gober, A.E. Increased recovery of Moraxella catarrhalis and Haemophilus influenzae in association with group A $\beta$ haemolytic streptococci in healthy children and those with pharyngo-tonsillitis. J. Med. Microbiol. 2006, 55, 989-992. [CrossRef] [PubMed]

38. Kim, S.W.; Lee, J.S.; Park, S.B.; Lee, A.R.; Jung, J.W.; Chun, J.H.; Lazarte, J.M.S.; Kim, J.; Seo, J.S.; Kim, J.H.; et al. The importance of porins and beta-lactamase in outer membrane vesicles on the hydrolysis of beta-lactam antibiotics. Int. J. Mol. Sci. 2020, $21,2822$. [CrossRef]

39. Marchant, P.; Carreño, A.; Vivanco, E.; Silva, A.; Nevermann, J.; Otero, C.; Araya, E.; Gil, F.; Calderón, I.L.; Fuentes, J.A. “One for All": Functional transfer of OMV-mediated polymyxin B resistance from Salmonella enterica sv. Typhi $\Delta t o l R$ and $\Delta d e g S$ to susceptible bacteria. Front. Microbiol. 2021, 12, 672467. [CrossRef] [PubMed]

40. Rumbo, C.; Fernández-Moreira, E.; Merino, M.; Poza, M.; Mendez, J.A.; Soares, N.C.; Mosquera, A.; Chaves, F.; Bou, G. Horizontal transfer of the OXA-24 carbapenemase gene via outer membrane vesicles: A new mechanism of dissemination of carbapenem resistance genes in Acinetobacter baumannii. Antimicrob. Agents Chemother. 2011, 55, 3084-3090. [CrossRef] [PubMed]

41. Chatterjee, S.; Mondal, A.; Mitra, S.; Basu, S. Acinetobacter baumannii transfers the $b l a_{\mathrm{NDM}-1}$ gene via outer membrane vesicles. J. Antimicrob. Chemother. 2017, 72, 2201-2207. [CrossRef]

42. Kosgodage, U.S.; Matewele, P.; Mastroianni, G.; Kraev, I.; Brotherton, D.; Awamaria, B.; Nicolas, A.P.; Lange, S.; Inal, J.M. Peptidylarginine deiminase inhibitors reduce bacterial membrane vesicle release and sensitize bacteria to antibiotic treatment. Front. Cell. Infect. Microbiol. 2019, 9, 227. [CrossRef]

43. Sauvage, E.; Terrak, M. Glycosyltransferases and transpeptidases/penicillin-binding proteins: Valuable targets for new antibacterials. Antibiotics 2016, 5, 12. [CrossRef] [PubMed] 